\title{
A novel prognostic prediction model for recurrence in patients with stage II colon cancer after curative resection
}

\author{
KAZUHIRO SASO ${ }^{1}$, NORIKATSU MYOSHI ${ }^{1,2}$, SHIKI FUJINO $^{1}$, YUYA TAKENAKA ${ }^{2}$, YUSUKE TAKAHASHI ${ }^{2}$, \\ JUNICHI NISHIMURA ${ }^{2}$, MASAYOSHI YASUI ${ }^{2}$, MASAYUKI OHUE $^{2}$, MASAYOSHI TOKUOKA $^{3}$, \\ YOSHITO IDE ${ }^{3}$, HIDEKAZU TAKAHASHI ${ }^{1}$, NAOTSUGU HARAGUCHI ${ }^{1}$, TAISHI HATA ${ }^{1}$, \\ CHU MATSUDA $^{1}$, TSUNEKAZU MIZUSHIMA ${ }^{1}$, YUICHIRO DOKI $^{1}$ and MASAKI MORI ${ }^{1}$ \\ ${ }^{1}$ Department of Gastroenterological Surgery, Osaka University Graduate School of Medicine, Suita, \\ Osaka 565-0871; ${ }^{2}$ Department of Surgery, Osaka International Cancer Institute, Osaka, Osaka 537-8511; \\ ${ }^{3}$ Department of Surgery, Yao Municipal Hospital, Yao, Osaka 581-0069, Japan
}

Received April 16, 2018; Accepted October 2, 2018

DOI: $10.3892 /$ mco.2018.1733

\begin{abstract}
The present study aimed to clarify the risk factors for recurrence of stage II colon cancer in patients and to determine possible treatment options for postoperative adjuvant chemotherapy. A prediction model for recurrence in patients with stage II colon cancer after curative surgical resection was developed. The present study retrospectively investigated 436 patients who underwent curative resection for stage II colon cancer at Osaka International Cancer Institute and Yao Municipal Hospital between 2004 and 2012. Several clinicopathological factors were examined and the Cox regression model was used to develop a prediction model for recurrence. The prediction model was validated in an independent group of 213 patients who underwent surgery at Osaka University Hospital between 2001 and 2012. Univariate analysis revealed that preoperative serum carcinoembryonic antigen level, preoperative obstruction, tumor invasion, lymphatic invasion and venous invasion were significantly correlated with disease-free survival. Using these variables, a classification and regression tree was constructed as a prediction model. The prediction models were validated by external datasets in an independent patient group. The concordance indices for DFS after current surgical resection were 0.675 in the learning set and 0.552 in the validation set. To conclude, a novel, reliable and personalized prognostic model was developed to predict recurrence in patients with stage II colon cancer, which may help clinicians to determine and perform adjuvant chemotherapy.
\end{abstract}

Correspondence to: Dr Norikatsu Miyoshi, Department of Gastroenterological Surgery, Osaka University Graduate School of Medicine, 2-2 Yamadaoka, Suita, Osaka 565-0871, Japan

E-mail: nmiyoshi@gesurg.med.osaka-u.ac.jp

Key words: stage II colon cancer, prediction model, CART, adjuvant chemotherapy, recurrence

\section{Introduction}

Colorectal cancer (CRC) is a frequent cause of cancer-related mortality (1) and is estimated to be the second leading cause of cancer-related death for both males and females (2). Lately, the incidence rate of CRC has been increasing in developing countries as well $(3,4)$. Although cancer stage is the major prognostic factor for CRC, the actual risks of recurrence are variable in individuals, even if they are at the same stage. Reportedly, there is a subset of patients with stage II colon cancer who have a high likelihood of recurrence and have attained a clinically significant benefit from chemotherapy (5). However, few studies have examined to define the subset of patients with stage II colon cancer who are at high risk of recurrence. While the American Society of Clinical Oncology guidelines do not recommend routine adjuvant chemotherapy for stage II patients (6), the European Society of Medical Oncology (ESMO) guidelines recommended that adjuvant chemotherapy be used for selected patients with stage II CRC. Hence, the necessity of adjuvant treatment at stage II is controversial.

Various studies have reported that factors such as T4 disease, poorly differentiated histology, tumor perforation, vascular invasion, lymphatic invasion, neuroinvasion, perineural invasion, bowel obstruction, surgical margin positivity, and inadequate lymph node sampling are related with poor prognosis in stage II CRC $(3,5)$.

According to data from the Surveillance, Epidemiology, and End Results (SEER) program in the United States, some reports have suggested that the prognosis of stage IIB (T4, N0) patients is worse than that of stage IIIA (T1-2, N1) patients (7). In recent years, these predictive factors for high-risk colon cancer have been retrospectively evaluated, and it is challenging to determine the benefits after curative resection and predict the prognosis in patients with stage II CRC $(8,9)$.

In this study, we report a prediction model that can predict recurrence of stage II colon cancer in patients after curative resection. Furthermore, it provides a prediction probability for individual outcomes that will benefit patients in selecting adjuvant treatment after curative resection. 


\section{Patients and methods}

Patients and dataset. In this study, 436 patients at Osaka International Cancer Institute and Yao Municipal Hospital were identified as having a diagnosis of stage II colorectal cancer from July 2004 to September 2012. All patients were histologically diagnosed with colorectal cancer and underwent curative surgical resection. A total of 84 patients with tumors located in the upper and lower rectum and/or who underwent adjuvant chemotherapy and neoadjuvant chemotherapy were excluded, and the remaining 352 patients were included. This study was approved by the Institutional Review Boards of our institutes. Patient records were anonymized before the analysis.

These 352 patients were included in the learning set, and the Cox proportional hazards regression model was used to develop the prediction model for the 5-year disease-free survival (DFS). An independent group of 213 patients who underwent curative resection for stage II colon cancer at Osaka University Hospital from January 2001 to December 2012 was used to validate the prediction model.

Clinicopathological evaluation and follow-up. Data on the age, sex, body mass index (BMI), preoperative serum level of the tumor marker carcinoembryonic antigen (CEA), pre-perforation, pre-obstruction, post-anastomotic leakage, surgery, dissection for lymph node, number of lymph nodes sampled, colorectal tumor location, and pathological findings (e.g., histology, histological grade, tumor invasion, lymph node sampling, lymphatic invasion, and venous invasion) were retrieved from patients' medical records for evaluation. Preoperative determination of the extent of tumor spread was performed using X-ray, CT, MRI, and/or positron emission tomography. Preoperative obstruction was defined as intestinal stenosis diagnosed by endoscopy and the starvation was necessary for over a week until colorectomy. Intraoperative findings contributed to the determination of metastatic tumor involvement. Surgical specimens were fixed in formalin, processed through a graded ethanol series, and embedded in paraffin. The sections were stained with hematoxylin and eosin and Elastica van Gieson stain, and the histological grade, degree of lymphatic invasion, and degree of venous invasion were examined. The clinicopathological factors were assessed according to the TNM classification of the UICC (10). After surgery, all patients underwent follow-up blood examinations to evaluate the serum levels of the tumor markers CEA and carbohydrate antigen 19-9, and further imaging with abdominal ultrasonography, CT, chest X-ray, and/or positron emission tomography every 3-6 months and colonoscopy every year following the Japanese guidelines (11).

Statistical analysis. Univariate analysis was performed using a proportional hazards model for DFS after primary curative resection to identify the independent factors. The two-sided $\mathrm{P}<0.05$ were considered statistically significant. A classification and regression tree (CART) was structured as a prediction model for DFS by variables, with the limit to enter a variable in the analysis set at $\mathrm{P}<0.05$. The Kaplan-Meier survival curves were plotted and compared
Table I. Clinicopathological factors in 352 patients with stage II colorectal cancer.

\begin{tabular}{lc}
\hline Clinicopathological factors & $\mathrm{N}=352$ \\
\hline Age (years) & $69(34-96)^{\mathrm{a}}$ \\
Sex (male/female) & $196 / 156$ \\
BMI & $22.7(12.1-49.9)^{\mathrm{a}}$ \\
CEA (ng/ml) & $2.5(0.1-296.1)^{\mathrm{a}}$ \\
Pre-obstruction (present/absent) & $12 / 340$ \\
Pre-perforation (present/absent) & $4 / 348$ \\
Post-anastomosis leakage (present/absent) & $8 / 344$ \\
Surgery (open/laparoscopy) & $290 / 62$ \\
Lymph node dissection ${ }^{\mathrm{b}}$ (D1,2/D3) & $63 / 289$ \\
Number of lymph nodes sampling & $80 / 272$
\end{tabular}

$(<12 / \geq 12)$

Tumor location

$32 / 77 / 45 / 30 / 120 / 48$

(Caecum/ascending/transverse/

descending/sigmoid/rectosigmoid)

Tumor invasion ${ }^{\mathrm{c}}\left(\mathrm{T} 3 / \mathrm{T} 4^{\mathrm{b}} / \mathrm{T} 4^{\mathrm{d}}\right)$

$295 / 44 / 13$

$332 / 20$

$132 / 220$

$205 / 147$

Lymphatic invasion (present/absent)

Venous invasion (present/absent)

Recurrence (present/absent)

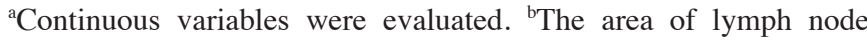
dissection defined in the Japanese Classification of Colorectal

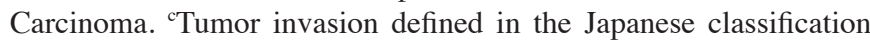
of colorectal carcinoma. ${ }^{\mathrm{d}}$ Well and moderately differentiated adeno-

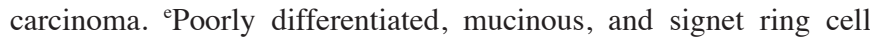
adenocarcinoma. CEA, carcinoembryonic antigen; BMI, body mass index.

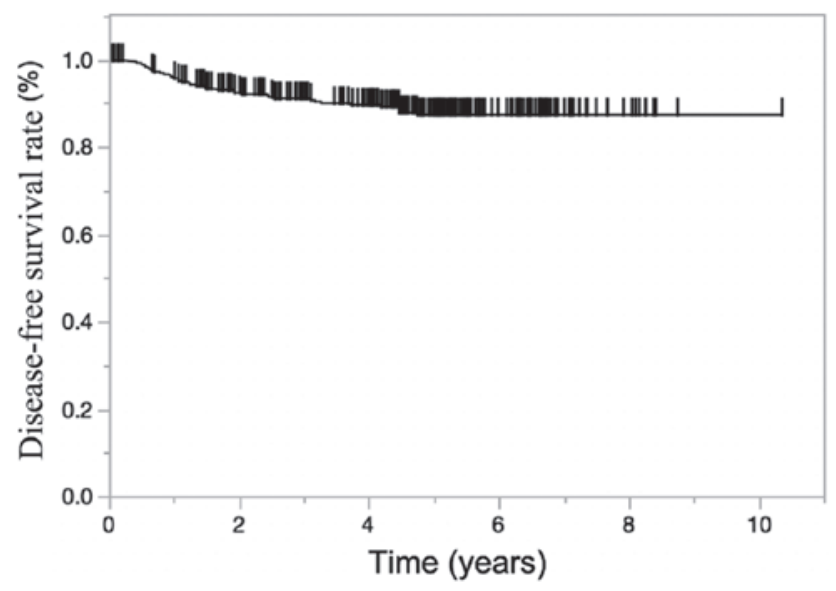

Figure 1. Kaplan-Meier survival curve for disease-free survival in patients with stage II colon cancer. The curve was plotted and compared with the generalized log-rank test. The median follow-up time was 4.67 years and the 5 -year disease-free survival was $89.2 \%$.

with the generalized log-rank test. All statistical analyses were performed using the JMP 13.0 statistical software program (SAS Institute, Cary, NC, USA). The CART was structured using R 3.1.3 (CRAN; R Foundation for Statistical Computing, Vienna, Austria). 
Table II. Univariate analysis for disease-free survival.

\begin{tabular}{|c|c|c|c|}
\hline Factors & HR & $95 \% \mathrm{CI}$ & P-value \\
\hline Age, years $(<69 / \geq 69)$ & 1.472 & $0.775-2.882$ & 0.239 \\
\hline Sex (male/female) & 1.381 & $0.724-2.738$ & 0.331 \\
\hline BMI ( $\geq 22.7 /<22.7)$ & 1.374 & $0.725-2.660$ & 0.331 \\
\hline $\mathrm{CEA}(\geq 5.0 /<5.0)$ & 2.014 & $1.040-3.816$ & $0.038^{\mathrm{a}}$ \\
\hline Pre-obstruction (present/absent) & 5.211 & $1.782-12.220$ & $0.005^{\mathrm{a}}$ \\
\hline Surgery (open/laparoscopy) & 1.031 & $0.463-2.742$ & 0.945 \\
\hline Lymph nodes dissection $^{\mathrm{b}}$ (D3/D1-2) & 1.142 & $0.463-2.742$ & 1.142 \\
\hline Number of lymph node sampling $(<12 / \geq 12)$ & 1.286 & $0.600-3.179$ & 0.538 \\
\hline Histological grade (well-mod ${ }^{\mathrm{c}} /$ others $^{\mathrm{d}}$ ) & 2.415 & $0.524-42.861$ & 0.314 \\
\hline Tumor invasion ${ }^{\mathrm{e}}(\mathrm{T} 4 \mathrm{a}-\mathrm{b} / \mathrm{T} 3)$ & 2.485 & $1.076-5.305$ & $0.034^{\mathrm{a}}$ \\
\hline Lymphatic invasion (present/absent) & 2.036 & $1.076-3.910$ & $0.029^{\mathrm{a}}$ \\
\hline Venous invasion (present/absent) & 2.483 & $1.223-5.567$ & $0.011^{\mathrm{a}}$ \\
\hline Tumor location (left/right) & 1.123 & $0.889-3.540$ & 0.109 \\
\hline
\end{tabular}

${ }^{\mathrm{a}} \mathrm{P}<0.05$. ${ }^{\mathrm{b}} \mathrm{The}$ area of lymph node dissection as defined in the Japanese Classification of Colorectal Carcinoma. ${ }^{\mathrm{c}}$ Well and moderately differ-

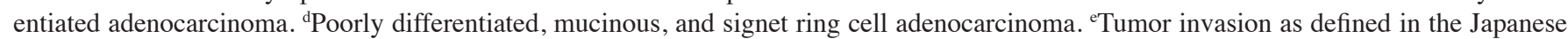
Classification of Colorectal Carcinoma. HR, hazard ratio; CI, confidence interval; BMI, body mass index.

\section{Results}

The characteristics of all 352 patients in the learning set are listed in Table I. All patients' ages ranged from 34 to 96 years, and 196 patients $(55.7 \%)$ were male. The median follow-up time was 4.67 years, and the 5-year DFS rate was $89.2 \%$ (Fig. 1). After curative surgical resection, 314 patients (89.2\%) had no recurrence. The univariate analysis of the clinicopathological factors for recurrence (Table II) revealed that high preoperative serum CEA level $(\mathrm{P}=0.038)$, pre-obstruction $(\mathrm{P}=0.005)$, tumor invasion $(\mathrm{P}=0.034)$, lymphatic invasion $(\mathrm{P}=0.029)$, and venous invasion $(\mathrm{P}=0.011)$ were significant factors for recurrence. These factors were introduced to create the prediction model as the CART. Finally, patients were segregated into six groups based on the following four factors: CEA level, tumor invasion, lymphatic invasion, and venous invasion. The DFS rates for each subgroup are shown in Figs. 2 and 3. The concordance indices for DFS after current surgical resection were 0.675 in the learning set and 0.552 in the validation set.

The CART divided the six groups based on the following four factors. Group 6 was the group with the highest risk of recurrence, and 5-year DFS was low at $68.6 \%$. Patients in group 6 gains more benefit from adjuvant chemotherapy than other groups.

\section{Discussion}

Although curative surgical resection is an effective treatment for CRC, some patients experience recurrence, which results in poor prognosis. In stage III, about $30 \%$ of patients experienced recurrence after curative resection $(8,9)$. The current guidelines recommend adjuvant chemotherapy for stage III CRC. Although recent chemotherapeutic development has improved the overall outcomes of advanced metastatic CRC, metastasis influences the prognosis. In stage II, about $13 \%$ of patients experience recurrence after curative resection, and the administration of adjuvant chemotherapy for patients with stage II CRC is controversial (12). The explorative CART analysis was used to define the relevant prognostic groups concerning survival probability. CARTs are machine-learning methods for constructing prediction models. Models are obtained by a recursive partitioning method using data, fitting a simple prediction model for each partition. Regression trees are for dependent variables taking continuous or discrete values, with prediction error measured by the squared difference between the observed and predicted values. During the CART analysis, first, the entire sample, and, after that, all newly defined subgroups were investigated at every stage of the analysis to determine which variable yielded the most significant division into the prognostic groups regarding the estimates of DFS probabilities according to Kaplan-Meier analysis and compared with log-rank test statistics (13).

Because clinical trials have not demonstrated a significant improvement in DFS, inclusion of a surgery-alone control arm in randomized trials for average-risk stage II patients remains justifiable. Meanwhile, the oncology research community has recently emphasized conducting trials to establish the role of molecular prognostic and predictive factors better. The motivation behind these trials is the identification of those patients who are most likely to benefit from treatment by their high risk of recurrence and/or high probability of response to treatment. As such, models to predict the prognosis after curative surgical resection would be useful in determining the necessity of an intensive follow-up to select adjuvant therapy. In our study, the clinicopathological analysis revealed that high preoperative serum CEA level, pre-obstruction, pathologically defined tumor invasion, lymphatic invasion, and venous invasion were associated with a poor prognosis for DFS.

Patients were divided into six groups by the CART based on the following four factors. Group 6 had the highest risk 


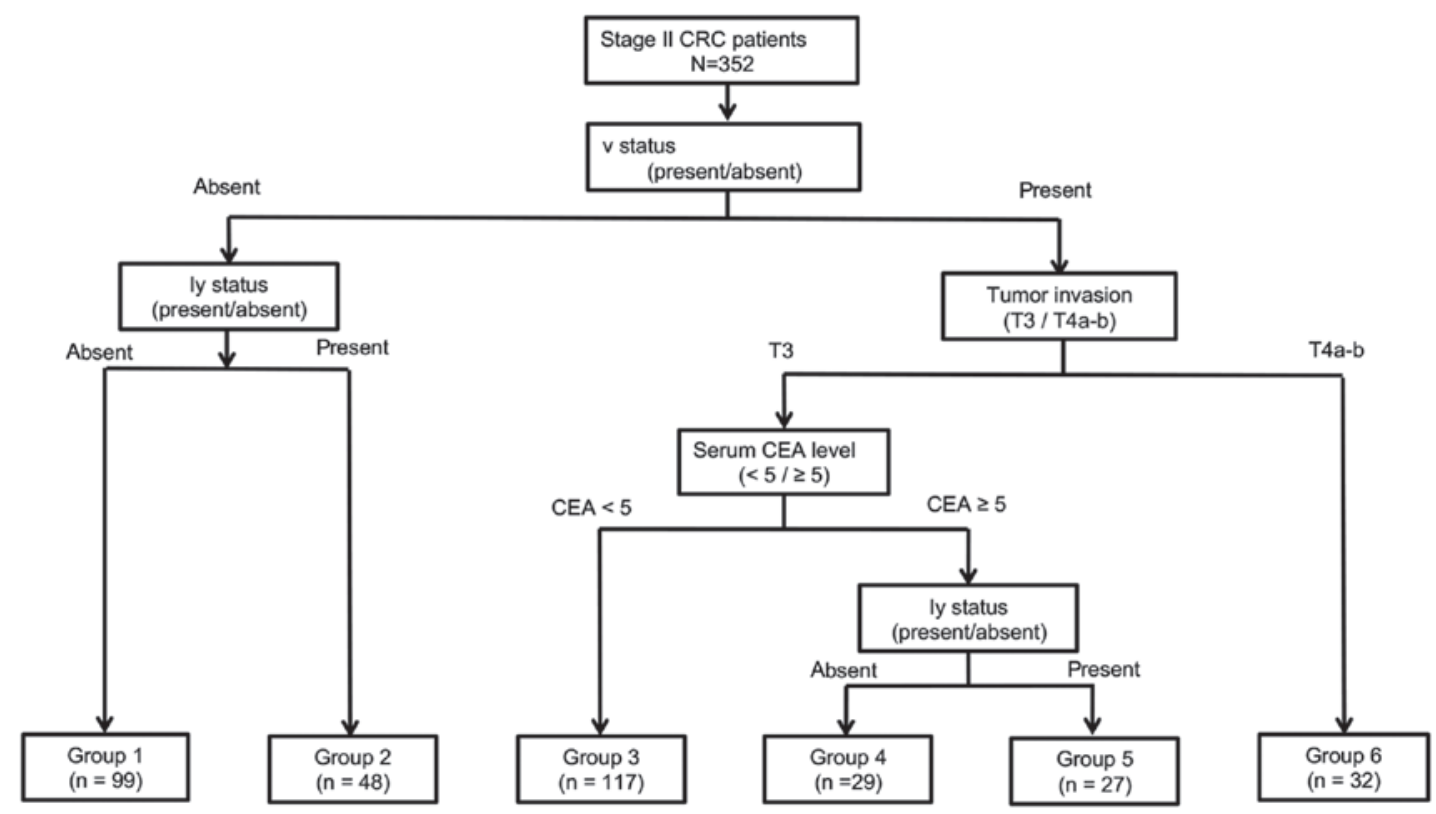

Figure 2. CART to predict disease-free survival after curative surgical resection for stage II colon cancer. Preoperative serum CEA level, tumor invasion, lymphatic invasion and venous invasion were used to classify subgroups. This tree was subjected to Kaplan-Meier analysis and compared with log-rank test statistics for recurrence of CRC, and was subsequently separated into six nodes. Each node suggested each other risk. CART, classification and regression tree; CEA, carcinoembryonic antigen; CRC, colorectal cancer; v status, venous invasion status; ly status, lymphatic invasion status.
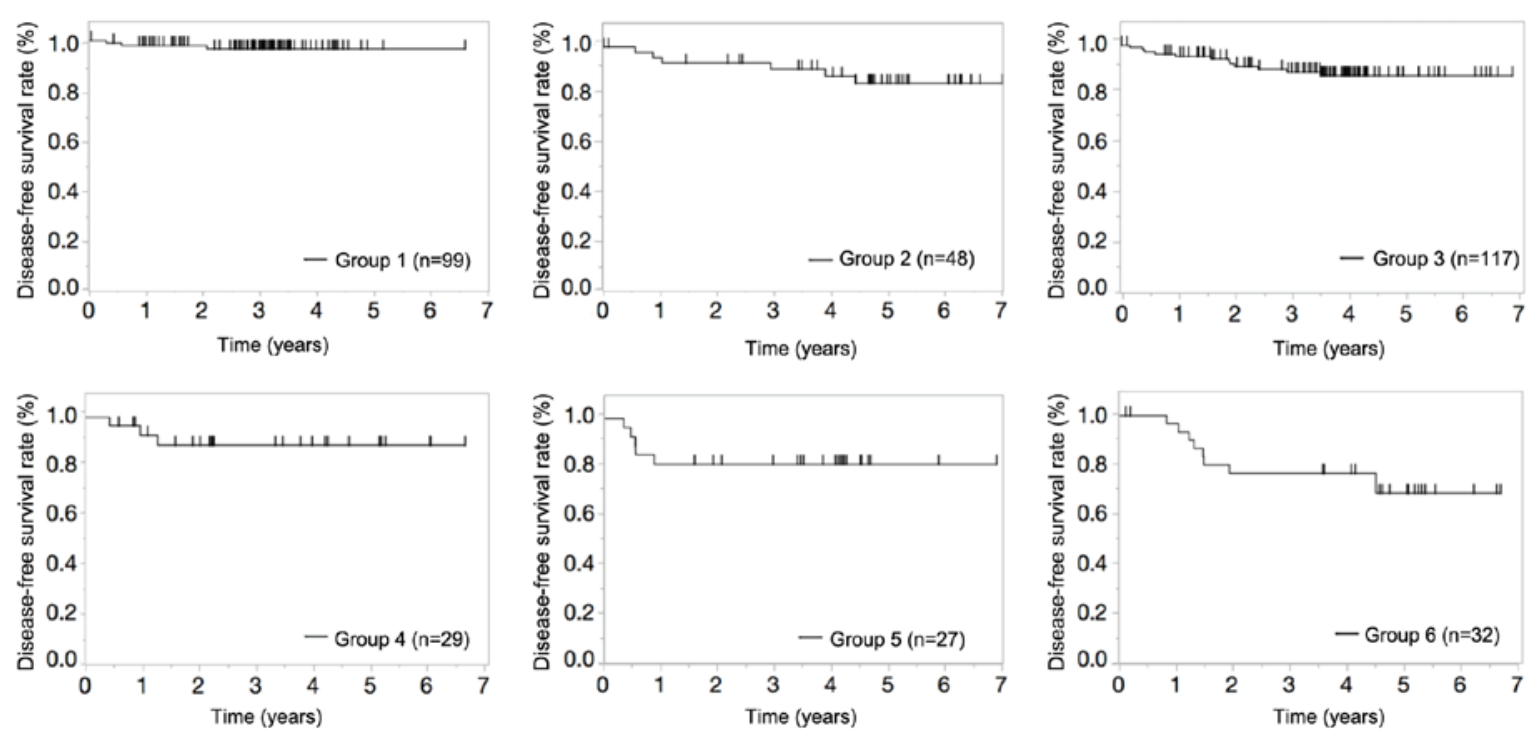

Figure 3. Kaplan-Meier survival curve for disease-free survival in subgroups. There were five subgroups and the 5-year disease-free survival rate was $96.6 \%$ in group $1,85.1 \%$ in group $2,87.8 \%$ in group $3,88.7 \%$ in group $4,81.3 \%$ in group 5 , and $68.6 \%$ in group 6 .

group of recurrence, with a 5-year DFS of $68.6 \%$. This recurrence rate is roughly the same as that in stage III patients after curative resection. We also analyzed patients who underwent adjuvant chemotherapy and were excluded from this study because there are influence of chemotherapy in DFS. As there was also concern that high risk groups of recurrence were excluded, we examined about the patients who underwent adjuvant chemotherapy in the DFS. The patients who underwent adjuvant chemotherapy classified in group 6 had no recurrence. This indicates that there are some patients who can benefit from adjuvant chemotherapy in stage II, and adjuvant chemotherapy might improve postoperative prognosis in the highest risk group.
We developed a prediction model for recurrence in patients with stage II colon cancer. High preoperative serum CEA level, pre-obstruction, tumor invasion, lymphatic invasion, and venous invasion were significant factors of recurrence in the univariate analysis for DFS and they constructed the branches of the CART. However, there are some limitations. Although preoperative obstruction was a significant factor in the univariate analysis, it was not included in the CART. We found there was a positive correlation between venous invasion and preoperative obstruction. Venous invasion appeared as the first branch of CART, and it might be a confounding factor for preoperative obstruction in our present retrospective analysis. We created the prediction model as a CART using five factors that were introduced by 
univariate analysis. We also performed the multivariate analysis to examine the predictive factors. There were only two significant factors, venous invasion $(\mathrm{P}=0.038)$ and pre-obstruction $(\mathrm{P}=0.029)$, in the multivariate analysis. Other factors that high preoperative serum CEA level $(\mathrm{P}=0.135)$, tumor invasion $(\mathrm{P}=0.172)$, and lymphatic invasion $(\mathrm{P}=0.097)$ were not significant for recurrence in the multivariate analysis. Two factors are too few to create the prediction model. Therefore, more patients and multi-institutional studies are necessary to create a universal CART from the results of multivariate analysis. Finally, some reported risk factors, such as poorly differentiated histology and bowel obstruction, were not significant, and tumor perforation, neuroinvasion, and perineural invasion were not examined in this study. Hence, a multiple-institutional study that enrolls many patients should be conducted.

However, the prediction model we have generated will help in selecting high-risk patients with stage II colon cancer who have significantly benefited from adjuvant chemotherapy. In addition, it will enable the selection of appropriate treatment for patients, resulting in effective outcomes.

In conclusion, a new prediction model was developed that indicates the risk of recurrence and prognosis in patients with stage II colon cancer, which will facilitate the appropriate introduction of adjuvant chemotherapy in high-risk stage II patients.

\section{Acknowledgements}

The authors would like to thank Ms. Aya Ito for special technical assistance regarding sample preparation (Department of surgery, Osaka International Cancer Institute, Osaka, Japan) for special technical assistance regarding sample preparation.

\section{Funding}

The present work was supported by a grant-in-aid for scientific research (grant no. 17K10572).

\section{Availability of data and materials}

All data generated or analyzed during this study are included in this published article.

\section{Authors' contributions}

KS and NM conceptualized the project, designed and performed the experiments and interpreted the results. KS also wrote the manuscript. NM, YT, YT, JN, MY, MO, MT, YI, HT, $\mathrm{NH}, \mathrm{TH}, \mathrm{TH}$ and $\mathrm{CM}$ performed the surgery and analyzed the clinical data. SF, NM, TM, YD and MM analyzed the data or participated in the discussions of the results.

\section{Ethics approval and consent to participate}

This study was approved by the Institutional Review Boards of our institutes. Patient records were anonymized before the analysis.

\section{Patient consent for publication}

Patients provided written, informed consent.

\section{Competing interests}

The authors declare that they have no competing interests.

\section{References}

1. Siegel R, Desantis C and Jemal A: Colorectal cancer statistics, 2014. CA Cancer J Clin 64: 104-117, 2014.

2. Siegel RL, Miller KD and Jemal A: Cancer statistics, 2017. CA Cancer J Clin 67: 7-30, 2017.

3. National Comprehensive Cancer Network: NCCN Clinical Practice Guidelines in Oncology: Colon Cancer. Version 2, 2017

4. Colvin H, Mizushima T, Eguchi H, Takiguchi S, Doki Y and Mori M: Gastroenterological surgery in Japan: The past, the present and the future. Ann Gastroenterol Surg 1: 5-10, 2017.

5. Labianca R, Nordlinger B, Beretta GD, Mosconi S, Mandala M, Cervantes A and Arnold D; ESMO Guidelines Working Group: Early colon cancer: ESMO clinical practice guidelines for diagnosis, treatment and follow-up. Ann Oncol 24 (Suppl 6): vi64-vi72, 2013.

6. Benson AB III, Schrag D, Somerfield MR, Cohen AM, Figueredo AT, Flynn PJ, Krzyzanowska MK, Maroun J, McAllister P, Van Cutsem E, et al: American Society of Clinical Oncology recommendations on adjuvant chemotherapy for stage II colon cancer. J Clin Oncol 22: 3408-3419, 2004.

7. O'Connell JB, Maggard MA and Ko CY: Colon cancer survival rates with the new American Joint Committee on Cancer sixth edition staging. J Natl Cancer Inst 96: 1420-1425, 2004.

8. Benson AB III, VenookAP, Cederquist L, Chan E, Chen YJ, Cooper HS, Deming D, Engstrom PF, Enzinger PC, Fichera A, et al: Colon Cancer, Version 1.2017, NCCN Clinical Practice Guidelines in Oncology. J Natl Compr Canc Netw 15: 370-398, 2017.

9. Kucukzeybek Y, Dirican A, Demir L, Yildirim S, Akyol M, Yildiz Y, Bayoglu IV, Alacacioglu A, Varol U, Salman T, et al: Adjuvant chemotherapy and prognostic factors in stage II colon cancer-Izmir Oncology Group Study. Asian Pac J Cancer Prev 16: 2413-2418, 2015.

10. Patriarca S, Ferretti S and Zanetti R: TNM Classification of malignant tumours-Eighth edition: Which news? Epidemiol Prev 41: 140-143, 2017 (In Italian).

11. Watanabe T, Muro K, Ajioka Y, Hashiguchi Y, Ito Y, Saito Y, Hamaguchi T, Ishida $\mathrm{H}$, Ishiguro $\mathrm{M}$, Ishihara $\mathrm{S}$, et al: Japanese Society for Cancer of the Colon and Rectum (JSCCR) guidelines 2016 for the treatment of colorectal cancer. Int J Clin Oncol 23: $1-34,2018$.

12. Wong AC, Stock S, Schrag D, Kahn KL, Salz T, Charlton ME, Rogers SO Jr, Goodman KA and Keating NL: Physicians' beliefs about the benefits and risks of adjuvant therapies for stage II and stage III colorectal cancer. J Oncol Pract 10: e360-e367, 2014.

13. Leiter U, Buettner PG, Eigentler TK and Garbe C: Prognostic factors of thin cutaneous melanoma: An analysis of the central malignant melanoma registry of the german dermatological society. J Clin Oncol 22: 3660-3667, 2004. 